\title{
Índice de massa corporal de adolescentes: comparação entre diferentes referências
}

\author{
Body mass index of adolescents: comparison among different references
}

Ana Paula G. Clemente ${ }^{1}$, Carla Danusa L. Santos², Ana Amélia Benedito-Silva ${ }^{3}$, Ana Lydia Sawaya

\section{RESUMO}

Objetivo: Comparar o desempenho das referências $(\mathrm{Na}$ tional Health and Nutrition Examination Survey I, National Center for Health Statistics/2000, International Obesity Task Force e Pesquisa Nacional sobre Saúde e Nutrição) para avaliação do estado nutricional de crianças e adolescentes à nova curva de valores de índice de massa corporal, proposta pela Organização Mundial da Saúde em 2007.

Métodos: Trata-se de um estudo transversal, cuja população foi constituída por uma amostra não probabilística, com 5.122 crianças e adolescentes de baixa renda. Foi realizado o cálculo do tamanho amostral visando identificar a associação entre alteração na pressão arterial e baixa estatura nos diferentes estados nutricionais. Compararam-se as diferentes referências para avaliação do estado nutricional. Foram aplicados os testes $\mathrm{Q}$ de Cochran, McNemar, qui-quadrado e índice Kappa para comparar as proporções e a concordância da classificação de baixo peso e excesso de peso com as diferentes referências.

Resultados: As cinco referências utilizadas no presente estudo apresentaram diferenças entre si. Tanto para meninos como para meninas, as prevalências estimadas de baixo peso pela referência da Pesquisa Nacional sobre Saúde e Nutrição foram bem menores que as outras referências. Quanto à prevalência de excesso de peso para os meninos, esta foi maior pela referência da Pesquisa Nacional sobre Saúde e Nutrição. Encontrou-se concordância excelente $(\mathrm{k}>0,75)$ entre a maioria das classificações para excesso de peso analisadas.
Porém, a concordância entre as classificações para baixo peso apresentou-se fraca $(\mathrm{k}<0,40)$.

Conclusões: A nova referência da Organização Mundial da Saúde é adequada para classificar os distúrbios nutricionais nos adolescentes brasileiros.

Palavras-chaves: padrões de referência; adolescente; índice de massa corporal; sobrepeso; desnutrição.

\section{ABSTRACT}

Objective: To compare the performance of the references (National Health and Nutrition Examination Survey I, National Center for Health Statistics/2000, International Obesity Task Force and Pesquisa Nacional sobre Saúde e Nutrição) to assess the nutritional status of children and adolescents with the new reference of body mass index values proposed by the World Health Organization in 2007.

Methods: Cross-sectional study that enrolled a nonprobabilistic sample of 5,122 children and adolescents of low socioeconomic status. In the matrix study, sample size was calculated in order to identify the association between blood pressure changes and stunting in different nutritional status. This interim report relates to the comparison among different references for assessment of nutritional status. Cochran's Q, McNemar, chi-square and Kappa statistics were used to compare the proportion of underweight and overweight by different references and the agreement among them.
Instituição: Universidade Federal de São Paulo (Unifesp), São Paulo, SP, Brasil

${ }^{1}$ Mestre em Endocrinologia Clínica pelo Programa de Pós-Graduação em Endocrinologia Clínica da Unifesp, São Paulo, SP, Brasil

2Doutora em Pediatria pelo Programa de Pós-Graduação em Pediatria e Ciências Aplicadas à Pediatria da Unifesp, São Paulo, SP, Brasil

${ }^{3}$ Doutora em Engenharia Elétrica pela Escola de Artes, Ciências e Humanidades da Universidade de São Paulo (USP), São Paulo, SP, Brasil ${ }^{4}$ Doutora em Nutrição pelo Departamento de Fisiologia da Unifesp; Professora do Departamento de Fisiologia da Unifesp, São Paulo, SP, Brasil

\section{Endereço para correspondência: \\ Ana Paula G. Clemente \\ Rua Botucatu, $862,2^{\circ}$ andar \\ CEP 04023-060 - São Paulo/SP \\ E-mail: ana.clemente@unifesp.br}

Fonte financiadora: Fundação de Amparo à Pesquisa do Estado de São Paulo (Fapesp), processo 06/56218-0

Conflito de interesse: nada a declarar 
Results: The five references used in this study showed differences between them. Both for boys as for girls, the estimated prevalence of underweight by the Brazilian reference is much smaller than with the other references. For overweight girls, a higher prevalence was detected by the Pesquisa Nacional sobre Saúde e Nutrição reference, followed by the World Health Organization/2007. There was an excellent agreement $(k>0.75)$ between the references regarding overweight, but the agreement among them regarding underweight was weak $(\mathrm{k}<0.40)$.

Conclusions: The new reference of World Health Organization/2007 is appropriated for classifying nutritional disorders in Brazilian teenagers.

Key-words: reference standards; adolescent; body mass index; overweight; malnutrition.

\section{Introdução}

A última Pesquisa de Orçamentos Familiares realizada no Brasil (2002-2003) verificou um aumento na prevalência de adolescentes com excesso de peso $(16,7 \%)$, comparada aos levantamentos anteriores (1974-1975: 3,9\% e 1989: $8,3 \%)$. Simultaneamente, constatou-se declínio na prevalência de adolescentes com déficit de estatura para idade (1974-1975: 33,5\%; 1989: 20,5\%; 2002-2003: 10,8\%) e de baixo peso (1974-1975: 4,8\%; 1989: 2,4\%; 2002-2003: $2,8 \%)$ ao longo dos três levantamentos ${ }^{(1)}$. Esse aumento da prevalência de excesso de peso em adolescentes demanda métodos práticos de diagnóstico que permitam a triagem de jovens em risco $^{(1-6)}$.

A avaliação do estado nutricional por meio da antropometria é bastante complexa em adolescentes, devido à grande variabilidade do crescimento e das dimensões corporais nessa faixa etária - variabilidade esta que depende do estado nutricional dos indivíduos, bem como do desempenho do crescimento nas idades anteriores e de fatores hormonais relacionados ao processo da maturação sexual ${ }^{(1,5,7,8)}$.

A Organização Mundial da Saúde (OMS), em 1995, sugeriu o uso de índice de massa corporal [IMC = peso $(\mathrm{kg}) /$ altura $\left(\mathrm{m}^{2}\right)$ ] para a triagem de adolescentes com sobrepeso e obesidade, por ser bem correlacionado à gordura corporal e de fácil obtenção, ter referências para comparar diferentes populações e ainda permitir uma continuidade do critério utilizado para avaliação de adultos ${ }^{(9)}$.

A comparação entre as diferentes referências é relevante para área de saúde pública diante da diversidade de referências apontada na literatura e das divergências quanto às mais indicadas para essa fase de crescimento e desenvolvimento intenso. Há ainda as diferenças individuais e étnicas nas etapas do desenvolvimento puberal que dificultam a interpretação de índices e indicadores antropométricos.

O objetivo do presente trabalho foi avaliar o desempenho de diferentes critérios de avaliação do estado nutricional de pré-adolescentes e adolescentes com a nova curva de valores de IMC proposta pela OMS em 2007.

\section{Método}

Trata-se de um estudo transversal, cuja população foi constituída por uma amostra não probabilística com 5.122 crianças e adolescentes de baixa renda, de ambos os sexos e com idade entre sete e 19 anos, frequentadores de escolas de ensino fundamental da rede pública do quinto ao nono ano do ensino fundamental. Foi realizado cálculo do tamanho amostral visando identificar a associação entre alteração na pressão arterial (variável dependente) e baixa estatura (variável independente) nos diferentes estados nutricionais, por meio da estatística Odds Ratio (OR). Porém, no presente estudo, compararam-se as diferentes referências para avaliação do estado nutricional. Para tanto, foram feitos mutirões de antropometria em 13 escolas públicas no período de abril de 2006 a outubro de 2007, com a intenção de cobrir o universo de indivíduos matriculados. A perda amostral, por não comparecimento ou recusa, foi de 430 indivíduos (8\%) do universo de alunos matriculados.

Foram excluídos jovens com diagnóstico de doenças crônicas que interferissem diretamente no peso e na estatura e aqueles que, no momento da avaliação, tivessem alguma limitação física para obtenção das medidas antropométricas. Investigou-se também a possibilidade de haver meninas grávidas, o que foi critério de exclusão do estudo. A coleta de dados foi realizada por duas nutricionistas treinadas.

Para a avaliação antropométrica, aferiram-se peso e estatura conforme as normas recomendadas por Lohman et $a^{(10)}$, utilizando-se balança eletrônica (modelo SD-150, Country Technologies, Willisnton, Gay Mills, USA) com capacidade de $150 \mathrm{~kg}$ e precisão de $100 \mathrm{~g}$. A estatura foi avaliada com o uso de um estadiômetro padrão (modelo Alturexata), escolhendo-se o valor mais próximo de $0,1 \mathrm{~cm}$. Os alunos foram avaliados com o mínimo de roupas e descalços.

Para a classificação nutricional, foi empregado o IMC para idade e sexo, e as curvas utilizadas foram: National Health and Nutrition Examination Survey I (NHANES I) ${ }^{(11)}$, 
National Center for Health Statistics/2000 (NCHS/2000) ${ }^{(12)}$, International Obesity Task Force (IOTF) ${ }^{(7)}$, Pesquisa Nacional sobre Saúde e Nutrição (PNSN) ${ }^{(8)}$ e Organização Mundial da Saúde/2007 (OMS/2007)(13). A Tabela 1 mostra a descrição dos pontos de cortes para a classificação de baixo peso e excesso de peso, de acordo com as referências usadas no estudo.

A análise do estado nutricional foi realizada por meio do software Epi-Info, versão 3.3.2 para a referência $\mathrm{NCHS} / 2000^{(12)}$. Para as referências da $\operatorname{IOTF}^{(7)}, \mathrm{PNSN}^{(8)}$ e OMS $/ 2007^{(13)}$ utilizaram-se as respectivas tabelas com valores críticos propostos para cada sexo e idade. $\mathrm{Na}$ análise estatística, utilizou-se o software Statistical Package for the Social Sciences (SPSS) versão 16. Aplicou-se o teste Q de Cochran para comparar a proporção de baixo peso e excesso de peso na amostra total entre as referências estudadas. O teste de McNemar foi utilizado sempre que o Q de Cochran foi significativo para a comparação das referências NHANES I, NCHS/2000, IOTF, PNSN à nova referência da OMS/2007. $\mathrm{O}$ teste do qui-quadrado comparou as proporções entre os sexos de baixo peso e excesso de peso nas cinco referências analisadas. Por último, foi calculado o índice Kappa para verificar a concordância entre as diferentes referências e a curva da OMS/2007. Essa medida de concordância tem como valor máximo 1, que representa concordância total, e como valor mínimo 0 , indicando nenhuma concordância. Valores até 0,40 significam concordância fraca; entre 0,40 e 0,75, concordância razoável; e valores acima de 0,75 , concordância excelente $^{(14)}$. Foi definido em $5 \%(p<0,05)$ o nível de significância para todas as análises.
O estudo foi aprovado pelo Comitê de Ética em Pesquisa da Universidade Federal de São Paulo (Unifesp), observandose o cumprimento dos princípios contidos na Declaração de Helsinki.

\section{Resultados}

A Tabela 2 mostra a prevalência de baixo peso e excesso de peso segundo as referências NHANES I, NCHS/2000, IOTF, PNSN e OMS/2007. Observou-se diferença significante na prevalência de baixo peso entre os sexos para todas as referências utilizadas: em todas o baixo peso foi maior entre os meninos, com exceção da referência PNSN, na qual o baixo peso foi maior entre as meninas. Por outro lado, a prevalência de excesso de peso foi significantemente maior nas meninas quando utilizadas as referências PNSN e IOTF, enquanto não houve diferença entre os sexos para as demais referências.

A Tabela 2 mostra ainda a diferença estatística entre a prevalência de baixo peso e excesso de peso nas referências adotadas e a nova referência preconizada pela OMS/2007. Da mesma forma, a prevalência de excesso de peso é maior quando é adotada a referência OMS/2007 em relação às demais, com exceção da referência PNSN, cuja prevalência é bastante superior à OMS/2007.

A Figura $1 \mathrm{~A}$ mostra a prevalência de baixo peso segundo a faixa etária com as diferentes referências utilizadas. Observou-se um aumento na prevalência de baixo peso na faixa etária de 7-8 anos em relação à de 9-11 anos em todos os padrões estudados, com exceção da referência brasileira que

Tabela 1 - Pontos de corte para classificação de baixo peso e excesso de peso de acordo com as referências utilizadas no estudo

\begin{tabular}{|c|c|c|c|}
\hline Indicador & Referências & Variável & Ponto de corte \\
\hline \multirow{6}{*}{ Baixo peso } & NHANES $\mathbf{I}^{(10)}$ & IMC para sexo e idade & $<\mathrm{P} 5$ \\
\hline & $\mathrm{NCHS} / 2000^{(11)}$ & IMC para sexo e idade & $<$ P5 \\
\hline & $\operatorname{IOTF}^{(7)}$ & Não classifica & \\
\hline & $\mathrm{PNSN}^{(8)}$ & IMC para sexo e idade & $<17,5 \mathrm{~kg} / \mathrm{m}^{2}$ (aos 20 anos) \\
\hline & $\mathrm{OMS} / 2007^{(12)}$ & IMC para sexo e idade & $<17,2 \mathrm{~kg} / \mathrm{m}^{2}$ (feminino, aos 19 anos) \\
\hline & & & $<18,2 \mathrm{~kg} / \mathrm{m}^{2}$ (masculino, 19 anos) \\
\hline \multirow[t]{6}{*}{ Excesso de peso } & NHANES I(10) & IMC para sexo e idade & $\geq P 85$ \\
\hline & $\mathrm{NCHS} / 2000^{(11)}$ & IMC para sexo e idade & $\geq \mathrm{P} 85$ \\
\hline & $\operatorname{IOTF}^{(7)}$ & IMC para sexo e idade & $>25$ (aos 18 anos) \\
\hline & $\mathrm{PNSN}^{(8)}$ & IMC para sexo e idade & $>25$ (aos 20 anos) \\
\hline & OMS/2007(12) & IMC para sexo e idade & $>25$ (feminino, aos 19 anos) \\
\hline & & & $>25,4$ (masculino, aos 19 anos) \\
\hline
\end{tabular}

(7)IOTF: International Obesity Task Force 2000; (8)PNSN: Conde et al 2006; (10)NHANES I: Must et al 1991; (11)NCHS/2000 = Kuczmarski et al 2000 ;

(12)OMS/2007 = Onis et al 2007; P: percentil; IMC: índice de massa corporal. 
Tabela 2 - Prevalência de baixo peso e excesso de peso separada por sexo, de acordo com as referências NHANES I, NCHS/2000, IOTF, PNSN e OMS/2007. Dados expressos em n (\%)

\begin{tabular}{lccccc}
\hline & NHANES I & NCHS/2000 & IOTF & PNSN & OMS/2007 \\
\hline Baixo peso & & & & & \\
$\quad$ Meninos & $191(7,5)$ & $184(7,2)$ & $\dagger$ & $11(0,4)$ & $240(9,4)$ \\
Meninas & $87^{*}(3,4)$ & $98^{*}(3,8)$ & $\dagger$ & $51^{*}(2,0)$ & $146^{*}(5,7)$ \\
\hline Excesso de peso & & & & & \\
Meninos & $475(18,6)$ & $443(17,4)$ & $386(15,1)$ & $561(22,0)$ & $497(19,5)$ \\
Meninas & $493(19,2)$ & $476(18,5)$ & $515^{*}(20,0)$ & $686^{*}(26,7)$ & $517(20,1)$ \\
\hline Total & & & & & \\
Baixo peso & $278^{\S}(5,4)$ & $282^{\S}(5,5)$ & $\dagger$ & $62^{\S}(1,2)$ & $386(7,5)$ \\
Excesso de peso & $986^{\S}(18,9)$ & $919^{\S}(17,9)$ & $901^{\S}(17,6)$ & $1247^{\S}(24,3)$ & $1014 \S(19,8)$ \\
\hline
\end{tabular}

${ }^{*} p<0,05$, difere dos meninos (teste do qui-quadrado); ${ }^{\S} p<0,05$, difere das demais referências (teste de McNemar); $†$ IOTF não classifica baixo peso. NHANES I(11): National Health and Nutrition Examination Survey I; NCHS/2000(12): National Center for Health Statistics/2000; IOTF(7): International Obesity Task Force; PNSN(8): Pesquisa Nacional sobre Saúde e Nutrição; OMS/2007: Organização Mundial da Saúde/2007.

Tabela 3 - Concordância entre as cinco referências adotadas, mensurada pelo índice Kappa, segundo sexo

\begin{tabular}{lcccc}
\hline & NHANES I e OMS/2007 & NCHS/2000 e OMS/2007 & IOTF e OMS/2007 & PNSN e OMS/2007 \\
\hline Baixo peso & & & & \\
Masculino & 0,88 & 0,84 & $\dagger$ & 0,07 \\
Feminino & 0,73 & 0,78 & $\dagger$ & 0,50 \\
Total & 0,82 & 0,82 & & 0,26 \\
Excesso de peso & & & 0,85 & 0,82 \\
Masculino & 0,96 & 0,93 & 0,96 & 0,91 \\
Feminino & 0,97 & 0,94 & 0,90 & 0,86 \\
Total & 0,97 & 0,93 & \\
\hline
\end{tabular}

†IOTF não classifica baixo peso; concordância excelente Kappa $\geq 0,75$; concordância razoável: $0,40 \leq$ Kappa $<0,75$; concordância fraca: Kappa<0,40.

apresentou médias muito baixas em todas as faixas etárias. Quando a referência OMS/2007 foi utilizada, observaramse prevalências superiores às outras três referências durante toda a puberdade, isto é, entre as faixas etárias 9-11 anos e 15-17 anos. A utilização do teste Q de Cochran permitiu mostrar diferença significante entre todas as referências e a OMS/2007 a partir dos nove anos de idade. Não foi possível realizar o teste Q de Cochran na faixa etária menor (7-8 anos) devido ao pequeno número de observações.

A Figura $1 \mathrm{~B}$ apresenta a prevalência de excesso de peso por faixa etária. Observou-se uma queda bem característica nessa prevalência com o aumento da idade. O critério do PNSN mostrou prevalência bem superior às outras quatro referências entre as faixas etárias de 7-8 e 15-17 anos. Enquanto as referências NCHS/2000 e IOTF apresentaram as menores prevalências entre 7-8 e 18-19 anos de idade, a referência da OMS/2007 apresentou prevalência alta de excesso de peso, superior à do NCHS/2000 e do IOTF, mas inferior à observada com o PNSN entre 9-17 anos. Verificou-se diferença significante das referências estudadas quando comparadas à OMS/2007, nas faixas etárias 9-11, 12-14 e 15-17 anos.

A Tabela 3 mostra a concordância entre as classificações avaliada pela estatística Kappa, com valores mínimos de 0,07 e máximos de 0,97. Nota-se que as concordâncias foram fracas para classificação de baixo peso no sexo masculino quando a referência PNSN foi utilizada. Com relação ao sexo feminino, observou-se concordância razoável, mas ainda relativamente mais baixa, com a referência PNSN. Por outro lado, o teste de concordância mostrou concordância excelente entre todas as referências estudadas para a classificação de excesso de peso. 


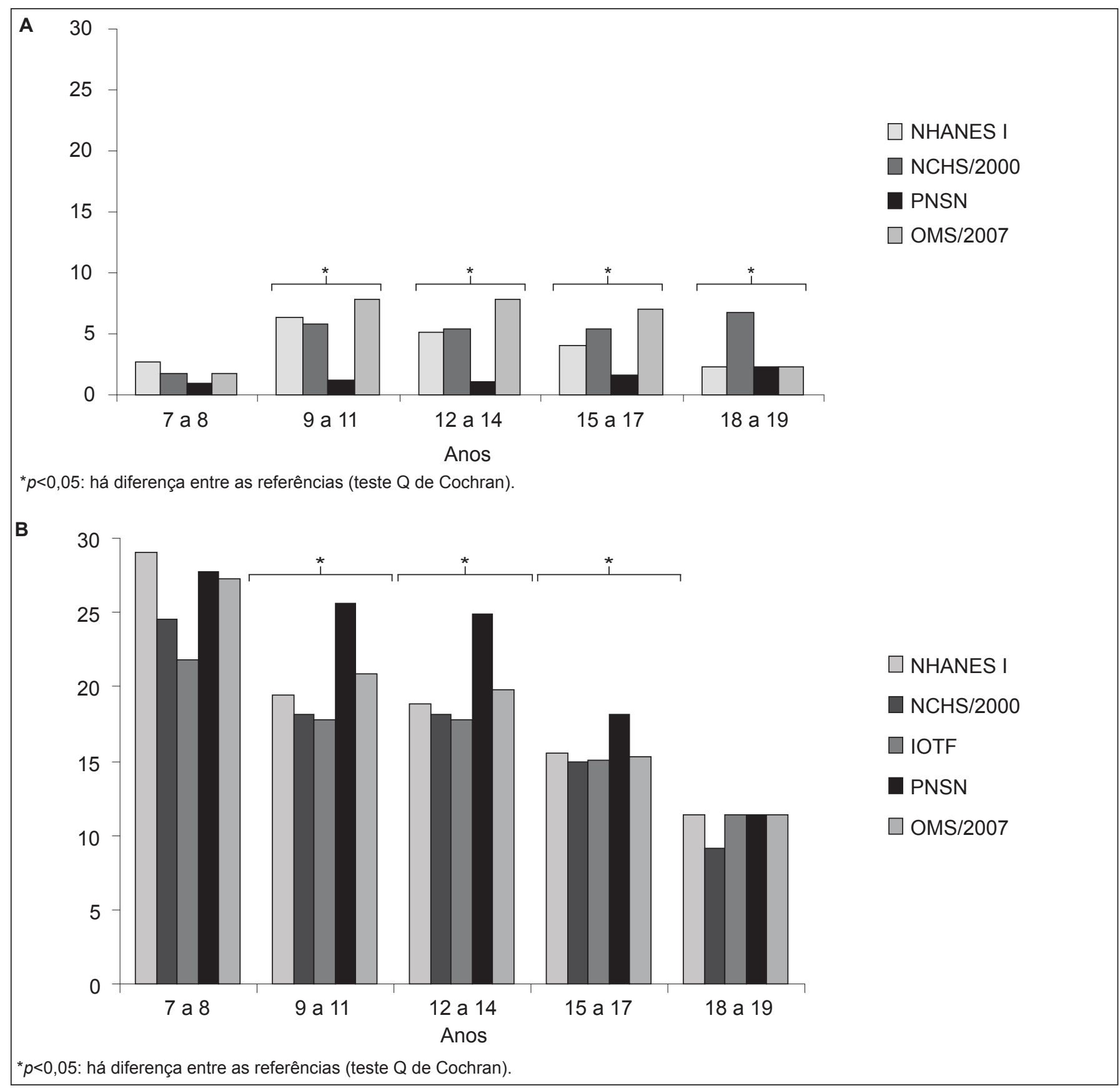

Figura 1 - Distribuição de baixo peso (A) e excesso de peso (B) de acordo com as referências NHANES I, NCHS/2000, IOTF, PNSN e OMS/2007, segundo faixas etárias.

\section{Discussão}

Estabelecer a melhor referência para avaliar o estado nutricional de adolescentes é uma tarefa bastante complexa. Em termos práticos, na perspectiva dos serviços de saúde, é desejável que se utilize um critério diagnóstico simples, replicável e confiável. A escolha de uma classificação adequada em serviços de saúde é importante, em primeiro lugar, para prevenção de distúrbios nutricionais no futuro e, em segundo lugar, para não sobrecarregar o serviço com a investigação e o tratamento de um grande número de falsos-positivos, rotulando equivocadamente muitos adolescentes normais como portadores de risco nutricional ${ }^{(3,15,16)}$.

Sabe-se que distúrbios nutricionais na infância e adolescência tendem a continuar na fase adulta se não forem adequadamente tratados, levando ao aumento da morbimortalidade 
e à diminuição da expectativa de vida ${ }^{(17,18)}$. Dessa forma, a detecção precoce de adolescentes com risco nutricional, assim como a adoção de medidas para controlar esse problema já na adolescência, faz com que o prognóstico seja mais favorável em longo prazo. Quanto mais tardia a detecção do distúrbio nutricional, mais difícil é a reversão deste, em função dos hábitos alimentares incorporados e das alterações metabólicas instaladas $^{(15,17)}$. Por esse motivo, na última década, surgiram várias referências para classificar o estado nutricional de adolescentes, o que gerou a necessidade de se realizarem comparações entre elas.

As cinco referências utilizadas no presente estudo apresentaram diferenças marcantes entre si. Tanto para os meninos como para as meninas, a prevalência estimada de baixo peso pela referência do PNSN é bem menor que a das outras referências. Observaram-se prevalências semelhantes de baixo peso quando as referências NHANES I e NCHS/2000 foram utilizadas, mas inferiores em relação à referência OMS/2007 em ambos os sexos. Em relação ao excesso de peso para meninas, observaram-se prevalências maiores pela referência PNSN, seguida pelas referências OMS/2007, IOTF, NHANES I e NCHS/2000. Quanto às prevalências de excesso de peso para os meninos, foi observada prevalência maior pela referência PNSN, seguida pelas referências OMS/2007, NHANES I, NCHS/2000 e por fim, IOTF.

As referências NCHS/2000, IOTF e OMS/2007, apesar de conterem, em parte, dados da mesma população norte-americana da referência NHANES I, apresentaram resultados diferentes para classificação de baixo peso e excesso de peso. O ponto de corte parece ser um dos fatores que diferenciou essas referências, tanto para o baixo peso como para o excesso de peso, influindo também o fato de alguns possuírem pontos de corte diferentes de acordo com o sexo ${ }^{(7,8,11-13)}$. Por outro lado, os novos ajustes matemáticos também devem ter contribuído para essas diferenças. Além disso, outro fator que pode ter provocado as diferenças nas prevalências encontradas quando se compararam as cinco referências no presente estudo estaria no aumento crescente

\section{Referências bibliográficas}

1. Instituto Brasileiro de Geografia e Estatística (IBGE). Pesquisa de orçamentos familiares 2002-2003: antropometria e análise do estado nutricional de crianças e adolescentes no Brasil. Rio de Janeiro: IBGE; 2006.

2. da Veiga GV, da Cunha AS, Sichieri R. Trends in overweight among adolescents living in the poorest and richest regions of Brazil. Am J Public Health 2004;94:1544-8. da obesidade na população americana, em particular, e também na Europa, ao longo das últimas décadas. As referências que contêm curvas com dados de peso obtidos após o aumento da obesidade ocorrido na década de 1980 podem estar subestimando a prevalência de obesidade e superestimando a prevalência de subnutrição, mesmo após ajustes para minimizar esse efeito ${ }^{(7,11,12)}$. Por esse motivo, as referências IOTF e OMS/2007 não utilizaram dados mais recentes de levantamentos antropométricos da população americana (1988-94) para a construção das curvas.

Quando se aplicou o teste Kappa para analisar a concordância entre as referências, encontrou-se concordância excelente $(k>0,75)$ entre a maioria das classificações para excesso de peso analisadas. Porém, a concordância entre as classificações para baixo peso mostrou-se fraca $(k<0,40)$, sobretudo no sexo masculino, quando foi realizada a seguinte comparação: PNSN versus OMS/2007.

Tomados em conjunto, os resultados dos diferentes critérios para aplicação do IMC para avaliar o estado nutricional de adolescentes, a partir da comparação das referências NHANES 1, NCHS/2000, IOTF, PNSN com a nova referência OMS/2007, mostraram, em primeiro lugar, uma boa concordância entre si para classificação de excesso de peso. Adicionalmente, quando a referência da OMS/2007 foi utilizada para classificação de baixo peso, encontraram-se valores superiores às demais referências e bem acima das prevalências encontradas para a referência brasileira. Assim, somada às vantagens do novo tratamento matemático, da possibilidade de se avaliar indivíduos de 0-19 anos em conjunto e da abrangência dos dados empíricos coletados em vários países utilizados para a construção da referência da OMS, encontrase a vantagem de ser uma referência única e mundial, que pode servir para comparar várias populações.

Por essas razões, os dados deste estudo indicam que a nova referência mostra-se adequada para classificar os distúrbios nutricionais nos adolescentes brasileiros e recomenda sua aplicação frente às outras classificações anteriormente existentes.

3. Vieira AC, Alvarez MM, de Marins VM, Sichieri R, da Veiga GV. Accuracy of different body mass index reference values to predict body fat in adolescents. Cad Saude Publica 2006;22:1681-90.

4. Veiga GV, Dias PC, dos Anjos LA. A comparison of distribution curves of body mass index from Brazil and United States for assessing overweight and obesity in Brazilian adolescents. Rev Panam Salud Publica 2001;10:79-85. 
5. Chiara V, Sichieri R, Martins PD. Sensitivity and specificity of overweight classification of adolescents, Brazil. Rev Saude Publica 2003;37: 226-31.

6. Abrantes MM, Lamounier JA, Colosimo EA. Comparison of body mass index values proposed by Cole et al. (2000) and Must et al. (1991) for identifying obese children with weight-for-height index recommended by the World Health Organization. Public Health Nutr 2003;6:307-11.

7. Cole TJ, Bellizzi MC, Flegal KM, Dietz WH. Establishing a standard definition for child overweight and obesity worldwide: international survey. BMJ 2000;320:1240-3.

8. Conde WL, Monteiro CA. Body mass index cutoff points for evaluation of nutritional status in Brazilian children and adolescents. J Pediatr (Rio J) 2006;82:266-72.

9. Autoria não referida. Physical status: the use and interpretation of anthropometry. Report of a WHO Expert Committee. World Health Organ Tech Rep Ser 1995;854:1-452.

10. Lohman TG, Roche AF, Martorell R. Antropometric standartization reference manual. Illinois: Human Kinetics Books; 1988.

11. Must A, Dallal GE, Dietz WH. Reference data for obesity: 85th and 95th percentiles of body mass index (wt/ht2) and triceps skinfold thickness. Am J Clin Nutr 1991;53:839-46.

12. Kuczmarski RJ, Ogden CL, Grummer-Strawn LM, Flegal KM, Guo SS, Wei R et al. CDC growth charts: United States. Advance data from vital and health statistics. Hyattsville (Maryland): National Center for Health Statistics; 2000.

13. de Onis M, Onyango AW, Borghi E, Siyam A, Nishida C, Siekmann J. Development of a WHO growth reference for school-aged children and adolescents. Bull World Health Organ 2007;85:660-7.

14. Agresti A. Categorical data analysis. New Jersey: Wiley; 2002.

15. Sotelo YO, Colugnati FA, Taddei JA. Prevalence of overweight and obesity in public school pupils according to three anthropometric diagnostic criteria. Cad Saude Publica 2004;20:233-40.

16. Wang Y, Moreno LA, Caballero B, Cole TJ. Limitations of the current world health organization growth references for children and adolescents. Food Nutr Bull 2006;27 (Suppl 4):S175-88.

17. Sawaya AL, Roberts S. Stunting and future risk of obesity: principal physiological mechanisms. Cad Saude Publica 2003;19 (Suppl 1):S21-8.

18. Must A. Morbidity and mortality associated with elevated body weight in children and adolescents. Am J Clin Nutr 1996;63:S445-7. 